\title{
Preference elicitation and learning
}

\author{
Vincent Mousseau ${ }^{1} \cdot$ Marc Pirlot $^{2}$
}

Published online: 22 May 2015

(C) Springer-Verlag Berlin Heidelberg and EURO - The Association of European Operational Research Societies 2015

Preferences are fundamental to decision processes, because decision analysts must account for the preferences of the stakeholders who participate in these processes and are impacted by the decision outcomes. To support the elicitation of stakeholder preferences, many models, procedures and methodologies have been proposed. These approaches to preference elicitation and learning will become more and more important with the proliferation of semi-automated computerized interfaces and the adoption of decision support systems which build on increasingly large datasets.

One of the major central tasks of the decision analyst is to elicit the judgements and value systems of the decision makers (DMs), including their views on the problem, and to integrate the resulting information into a preference model from which recommendations can be derived. This preference elicitation activity can be tricky: the preferences expressed by the DMs can be imprecise, conflicting, unstable, time-dependent, yet they should be structured and synthesized into numerical values (or intervals of numerical values) concerning the parameters that characterize preferences in the decision model.

For the domain Preference Elicitation and Learning, models, procedures and methodologies have been developed not only by researchers working in the field of Multiple Criteria Decision Aid but also in that of Artificial Intelligence. Their research has focused on the modeling, representation, elicitation, learning,

Vincent Mousseau

vincent.mousseau@ecp.fr

Marc Pirlot

marc.pirlot@umons.ac.be

1 Laboratoire Génie Industriel, Grande Voie des Vignes, Châtenay-Malabry, France

2 MATHRO, UMONS, Faculté Polytechniquede Mons, 9 Rue de Houdain, 7000 Mons, Belgium 
aggregation, and management of preferences. This work has led to the development of algorithms that can be embedded in methodological approaches to support different phases of the decision process, including the integration of the DM preferences, the elaboration of decision recommendations, and the understanding and acceptance of the decision recommendations by stakeholders.

Overall, the topic of Preference Elicitation and Learning has attracted plenty of interest as illustrated, for instance, by Fürnkranz and Hüllermeier (2010) and the activities of several EURO working groups (Multicriteria Decision Aid, Decision Support Systems, Advances in Preference Handling), Preference Learning streams at EURO conference, and numerous related workshops (ECAI and IJCAI Workshops, DA2PL). This vibrant research activity has lead to the development of new formalisms and methodologies and offered new perspectives into preference handling and elicitation. In particular, there have been significant advances on preference disaggregation and robust regression of preference (e.g., Doumpos and Zopounidis 2011; Greco et al. 2008), group preference elicitation (e.g., Cailloux et al. 2012; Greco et al. 2012), and explanation of decision recommendations (e.g., Labreuche 2011; Labreuche et al. 2012). Cases studies have offered insights into how preference learning and elicitation methodologies can be put into action to establish improved decision processes (Bisdorff et al. 2015).

This feature cluster contains three interesting papers which propose tools for the elicitation of preference models. These tools are intended to be used by analysts who seek to improve the relevance of the decision process for the DM. They share a common feature in that they build on relatively sophisticated preference models that represent non-independent preferences with interacting criteria. Such preferences cannot in general be represented by an additive value function (utility) model. Thus, the scores for ranking the alternatives in decreasing order of preference are, in general, computed as a Choquet integral of marginal value functions (utilities). The parameters for these models cannot be elicited using procedures for additive value functions, and consequently specific elicitation procedures are needed (see e.g., Grabisch et al. 2008). The three papers in this issue propose such procedures in different contexts in which the information required in the elicitation/learning process may require varying degrees of interaction with the DM or the possibility to obtain preference information through well-designed questions (active learning). Below we briefly outline the contributions in the three papers.

- The paper by Rolland et al. (2015) is devoted to the elicitation of preferences that can be represented by a bipolar Choquet integral of the alternatives' performances. Bipolar scales prove appropriate when the criterion scale has a natural "zero" value. Values below zero (losses) are perceived differently from those above zero (gains). The information required is a score for each alternative in a learning set. The model estimates the parameters of a bipolar Choquet integral that reproduces the known scores as accurately as possible.

- Benabbou et al. (2015) consider rank-dependent aggregation which integrates positive or negative synergies between criteria. They propose an incremental 
elicitation process in which each question is chosen to be as informative as possible, hence reduces the cognitive load on the DM.

- Labreuche et al. (2015) propose an interactive elicitation framework to estimate the parameters of an Ordered Weighted Average. This framework is analogous to the MACBETH methodology for the elicitation of an additive value model in that it asks the DM to make pairwise comparisons between binary alternatives which involve only two reference evaluation levels on each criterion.

\section{References}

Benabbou N, Gonzales C, Perny P, Viappiani P (2015) Minimax regret approaches for preference elicitation with rank-dependent aggregators. Eur J Decision Process. doi:10.1007/s40070-015-00406

Bisdorff R, Dias L, Meyer P, Mousseau V, Pirlot M (2015) Evaluation and decision models with multiple criteria: case studies. Springer, Berlin

Cailloux O, Meyer P, Mousseau V (2012) Eliciting ELECTRE TRI category limits for a group of decision makers. Eur J Oper Res 223(1):133-140

Doumpos M, Zopounidis C (2011) Preference disaggregation and statistical learning for multicriteria decision support: a review. Eur J Oper Res 209(3):203-214

Fürnkranz J, Hüllermeier E (2010) Preference learning. Springer, Berlin

Grabisch M, Kojadinovic I, Meyer P (2008) A review of methods for capacity identification in Choquet integral based multi-attribute utility theory: applications of the kappalab R package. Eur J Oper Res 186(2):766-785

Greco S, Kadzinski M, Mousseau V, Slowinski R (2012) Robust ordinal regression for multiple criteria group decision: UTAGMS-GROUP and UTADISGMS-GROUP. Decis Support Syst 52(3):549-561

Greco S, Mousseau V, Slowinski R (2008) Ordinal regression revisited: multiple criteria ranking using a set of additive value functions. Eur J Oper Res 191(2):415-435

Labreuche C (2011) A general framework for explaining the results of a multi-attribute preference model. Artif Intell 175(7-8):1410-1448

Labreuche C, Maudet N, Ouerdane W (2012) Justifying dominating options when preferential information is incomplete. In: Proceedings of the 20th European Conference on Artificial Intelligence (ECAI'12), vol 242 of Frontiers in Artificial Intelligence and Applications, IOS Press, 486-491

Labreuche C, Mayag B, Duqueroie B (2015) Extension of the MACBETH approach to elicit an ordered weighted average operator. Eur J Decision Process. doi:10.1007/s40070-015-0041-5

Rolland A, Ah-Pine J, Mayag B (2015) Elicitation of 2-additive bi-capacity parameters. Eur J Decision Process. doi:10.1007/s40070-015-0043-3 